\title{
Erhöhtes Schlaganfallrisiko bei Zoster ophthalmicus
}

Bei Patienten, die an Herpes zoster mit Gesichtsbeteiligung (Zoster ophthalmicus) erkrankt sind, ist auch an das Schlaganfallrisiko als mögliche Komplikation zu denken.

In dieser Beobachtungsstudie wurden 658 Patienten einbezogen, bei denen in den Jahren 2003 oder 2004 die Diagnose eines Zoster ophthalmicus gestellt wurde. Als Kontrollpatienten wurden 1974 Patienten (drei Kontrollen pro Fall, mit jeweils gleichem Alter und Geschlecht) ausgewählt, die im gleichen Zeitraum wegen anderer Erkrankungen behandelt wurden.

Innerhalb der einjährigen Nachbeobachtungszeit trat bei 8,1\% der Patienten mit Zoster ophthalmicus, aber nur bei $1,7 \%$ der alters- und geschlechtsgleichen Kontrollpatienten ein erstmaliger Schlaganfall auf $(\mathrm{p}<$ $0,001)$.

Die Analyse ergab, dass die 1-JahresRate erstmaliger Schlaganfälle bei Zosterpatienten im Vergleich zu den Kontrollpatienten um mehr als das Vierfache erhöht war (Hazard Ratio = 4,52).

\section{Kommentar}

Es wird geschätzt, dass ungefähr ein Drittel aller Menschen im Lauf ihres Lebens einen Herpes zoster durchmachen. Davon findet sich in bis zu $20 \%$ der Fälle eine Beteiligung von Gesicht und Augenbereich im Sinne eines Zoster ophthalmicus. Die vorliegende Studie zeigt, dass bei Patienten mit Zoster ophthalmicus auch an Schlaganfälle als wichtige Komplikation gedacht werden muss. Das Schlaganfallrisiko ist unabhängig vom Alter und

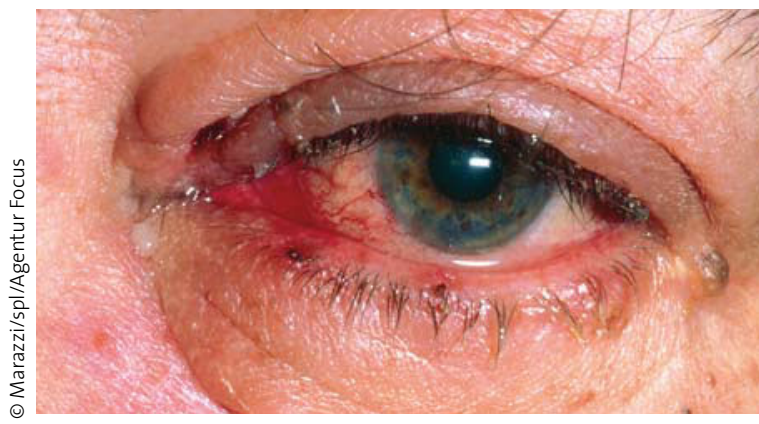

Zoster ophthalmicus.

von den vaskulären Risikofaktoren stark erhöht und zwar während des gesamten ersten Jahres nach der Zostererkrankung.

M. BUSCH, Robert KoCH-INStitut BerLin .

- H. C. Lin et al.

Herpes zoster ophthalmicus and the risk of stroke: a population-based follow-up study. Neurology 74 (2010) 792-797 